\title{
Homozygous SYNE1 mutation causes congenital onset of muscular weakness with distal arthrogryposis: a genotype-phenotype correlation
}

\author{
Matthias Baumann ${ }^{1}$, Elisabeth Steichen-Gersdorf ${ }^{1}$, Birgit Krabichler ${ }^{2}$, Britt-Sabina Petersen ${ }^{3}$, Ulrike Weber ${ }^{4}$, \\ Wolfgang M Schmidt ${ }^{5}$, Johannes Zschocke ${ }^{2}$, Thomas Müller ${ }^{1}$, Reginald E Bittner ${ }^{5}$ and Andreas R Janecke ${ }^{\star, 1,2}$ \\ The exceptionally large SYNE1 (spectrin repeat-containing nuclear envelope protein 1) gene encodes different nesprin-1 \\ isoforms, which are differentially expressed in striated muscle and in cerebellar and cerebral neurons. Nesprin-1 isoforms can \\ function in cytoskeletal, nuclear, and vesicle anchoring. SYNE1 variants have been associated with a spectrum of neurological \\ and neuromuscular disease. Homozygosity mapping combined with exome sequencing identified a disease-causing nonsense \\ mutation in the ultimate exon of full-length SYNE1 transcript in an 8-year-old boy with distal arthrogryposis and muscular \\ hypotonia. mRNA analysis showed that the mutant transcript is expressed at wild-type levels. The variant truncates nesprin-1 \\ isoforms for the C-terminal KASH (Klarsicht-ANC-Syne homology) domain. This is the third family with recessive arthrogryposis \\ caused by homozygous distal-truncating SYNE1 variants. There is a SYNE1 genotype-phenotype correlation emerging, with more \\ proximal homozygous SYNE1 variants causing recessive cerebellar ataxia of variable onset (SCAR8; ARCA-1).
}

European Journal of Human Genetics (2017) 25, 262-266; doi:10.1038/ejhg.2016.144; published online 26 October 2016

\begin{abstract}
INTRODUCTION
The SYNE1 (spectrin repeat-containing nuclear envelope protein 1) gene comprises $516 \mathrm{Mbp}$ on chromosome 6q25, contains 146 exons, and undergoes differential splicing. Nesprin-1 and nesprin-2 belong to the nesprin (nuclear envelope spectrin-repeat protein) family of nuclear and cytoskeletal proteins, and are expressed as different isoforms in a tissue-specific manner. Nesprins have a central rod domain composed of spectrin repeats that facilitate kinesin and dynein binding, and dimer formation. Full-length nesprin-1 and nesprin-2 isoforms have two N-terminal $\mathrm{CH}$ (calponin homology) domains that bind to the actin cytoskeleton and C-terminal KASH (Klarsicht-ANCSyne homology) domains, which reside in the outer nuclear membrane and bind to the SUN (Sad1p-UNC-84) domain proteins within the nuclear envelope lumen. Apart from full-length nesprin-1 (nesprin-1 giant, 8797 amino acids, $1 \mathrm{MDa}$ ), SYNE1 encodes at least $16 \mathrm{KASH}-$ and $14 \mathrm{CH}$-containing SYNE-1 isoforms. ${ }^{1-3}$ Of note, nesprin-1 $\alpha 2 \quad(120 \mathrm{kDa})$ represents a C-terminal, predominantly muscle-specific isoform, ${ }^{4}$ whereas KLNes1g represents a large KASH-less variant specifically expressed in the CNS and most abundant in the cerebellum. ${ }^{5}$

Disease-causing SYNE1 variants were previously reported in association with a spectrum of neuromuscular disease. We report a third family with arthrogryposis multiplex congenital (AMC) caused by a truncating SYNE1 variant and propose a SYNE1 genotype-phenotype correlation.
\end{abstract}

\section{CASE REPORT}

The boy reported here is the third child of healthy, first-grade consanguineous Turkish parents. His 11-year-old sister is healthy.
The first child of the family presented with severe muscular hypotonia at birth and died at the age of 4 months from respiratory failure. Pregnancy was normal, except for reduced fetal movements. The patient was born at 40 weeks of gestation by cesarean section. APGAR scores were $3 / 7 / 8$, respectively. The neonate was small for gestational age with a weight of $2464 \mathrm{~g}$ (below 3rd percentile), length of $46.5 \mathrm{~cm}$ (3rd percentile), and head circumference of $33.6 \mathrm{~cm}$ (30th percentile). Muscular weakness ('floppy infant'), adducted thumbs, flexion contractures of fingers, and clubfeet were noted at birth (Figure 1). Bilateral clubfeet were repaired at the age of 6 months and the adducted thumbs were splinted. Surgery for cryptorchidism was performed at 2.5 years and for left-sided clubfoot relapse at 5 years. He was able to walk unsupported at the age of 2 years. At the last visit at 8 years he showed marked proximal muscular weakness, reduced deep tendon reflexes, and mild facial weakness. There was neither pyramidal nor cerebellar involvement. Walking distance is $1 \mathrm{~km}$ or 10-15 min. He uses the Gowers maneuver to get up and he climbs 5 stairs, without alternating feet and using the stair railing. He has flexion contractures of the proximal interphalangeal joints of the third and fourth fingers (Figure 1). Hyperopia of +4 Dpt. with intermittent strabismus developed. Neuropsychological testing shows that his intellectual abilities are below average for his age. Growth deficiency increased despite sufficient weight gain. At present, his weight is at the 80th percentile, length below the 3rd percentile (SDS - 2.5) and head circumference at the 25th percentile.

Nerve conduction studies, electromyography, and brain, spine, heart and quadriceps muscle ultrasound showed normal results in the newborn period. Diagnostic multiplex ligation-dependent probe

${ }^{1}$ Department of Pediatrics I, Medical University of Innsbruck, Innsbruck, Austria; ${ }^{2}$ Division of Human Genetics, Medical University of Innsbruck, Innsbruck, Austria; ${ }^{3}$ Institute of Clinical Molecular Biology, Christian-Albrechts-University Kiel, Kiel, Germany; ${ }^{4}$ Department of Pediatrics, Krankenhaus Dornbirn, Dornbirn, Austria; ${ }^{5}$ Neuromuscular Research Department, Center for Anatomy and Cell Biology, Medical University of Vienna, Vienna, Austria

*Correspondence: Dr AR Janecke, Division of Human Genetics, Department of Pediatrics I, Medical University of Innsbruck, Anichstrasse 35, Innsbruck A-6020 Austria. Tel: +43 512504 23600; Fax: +43 512504 25450; E-mail: Andreas.Janecke@i-med.ac.at

Received 27 May 2016; revised 7 September 2016; accepted 20 September 2016; published online 26 October 2016 

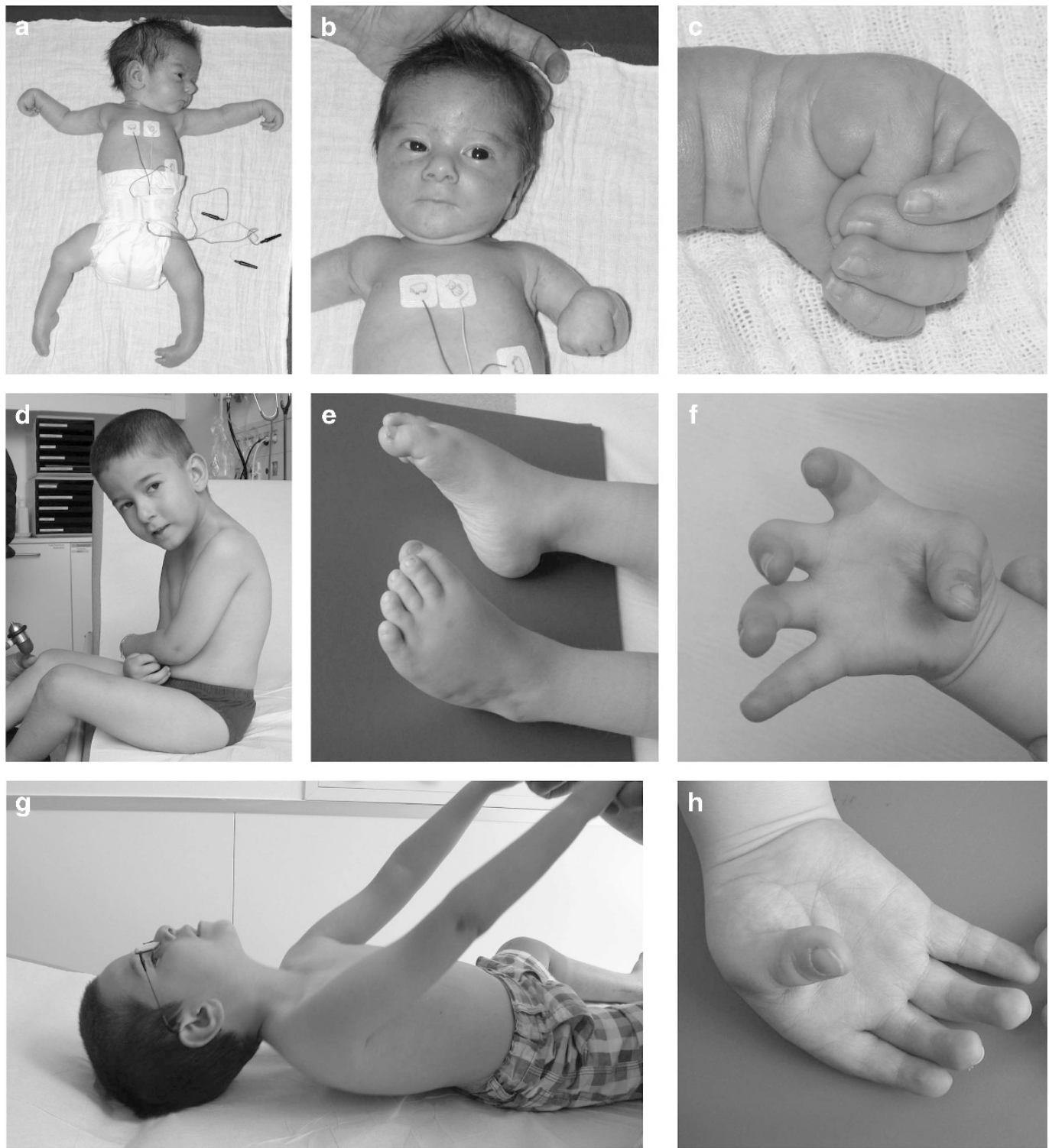

Figure 1 Clinical phenotype. Pictures taken at the ages of (a-c) 3 weeks, (d-f) 4.5 years, (g) 5.5 years, and (h) 7 years, showing distal contractures with adducted thumbs and clubfeet. At the age of 5.5 years, persisting muscular hypotonia and weakness are illustrated by head lag during traction response.

amplification analysis excluded the common variants underlying spinal muscular atrophy and chromosomal microarray analysis showed no abnormalities. Creatine kinase and cardiac troponin $\mathrm{T}$ and I were within normal limits. Electrocardiogram and echocardiography did not indicate cardiomyopathy and excluded abnormal conduction at the age of 7 years. Quadriceps muscle ultrasound revealed marked, diffusely increased muscle echo intensity, indicating replacement of healthy muscle tissue by fibrosis and fatty infiltration.

\section{MATERIALS AND METHODS}

Written informed consent for molecular research investigations was obtained from the patient's parents and the study was approved by the local ethics committee. Homozygosity mapping of the disease locus in the family and exome sequencing in the proband was performed as described in Supplementary Material. The SYNE1 variant designation is based on the NCBI reference sequence for transcript NM_182961.3 (corresponding to Ensembl transcript reference sequence ENST00000 367255) and the genomic reference sequence NG_012855.1. The exon numbering is based on NG_012855.1.

\section{RESULTS AND DISCUSSION}

Congenital distal arthrogryposis and muscular hypotonia occur in a large number of neuromuscular and connective tissue disorders. We identified a patient with recessive AMC, muscular hypotonia associated with delayed motor development, and with persistent contractures of interphalangeal joints. Exome sequencing identified a homozygous variant c.26236C $>\mathrm{T}$ causing a premature stop codon (p. $\left.\left(\operatorname{Arg} 8746^{*}\right)\right)$ in the ultimate exon of full-length SYNE1. This variant was submitted to the gene-specific database at http://databases.lovd.nl/shared/genes/SYNE1 (patient ID 00079635). This rare variant $(1 / 121366$ alleles in EXAC) segregates with disease in the family (Supplementary Figure S1). 
This is the third homozygous distal-truncating SYNE1 variant and all three have been associated with recessive AMC, in five patients reported to date. ${ }^{6,7}$ Apart from AMC, shared clinical findings in these patients are decreased fetal movements, absence of polyhydramnios, no marked intrauterine growth retardation, and the absence of associated malformations. Motor development is mildly delayed with borderline to normal intellectual development, without elevation of creatine kinase levels. The disease was progressive in a sib-pair from one family, who were unable to walk at the age of 12 years, and developed severe scoliosis, leading to death from pneumonia at 22 years in one patient. ${ }^{6}$ There is no indication of cerebellar disease in these patients. However, as three of four living patients are younger than 8 years at last reporting, no definite conclusions as to the course of the disease can be drawn at present.

Analysis of fibroblast-derived cDNA showed that the p. $\left(\operatorname{Arg} 8746^{*}\right)$ mutant transcript is produced at normal levels, indicating that normal quantities of nesprin-1 protein truncated for the C-terminal 50 amino acids are produced. In contrast, the AMC-associated homozygous splice site variant c.24313-2A $>$ G resulting in p.(His8105Valfs ${ }^{\star} 8$ ) was shown to reduce SYNE1 RNA stability and deplete nesprin-1 in patient fibroblasts. ${ }^{6}$ The same would be expected for the c.24577C $>\mathrm{T}$ (p. $\left.\left(\operatorname{Arg} 8193^{*}\right)\right)$ variant, identified in another sib-pair with AMC. ${ }^{7}$ Given the similar presentation of these five patients in their first years of life, this indicates that loss of the KASH domain is sufficient to cause SYNE1-associated AMC (Figure 2 and Supplementary Table S1). All three AMC-causing SYNE1 variants would be expected to affect at least the major muscle-specific, KASH-containing isoform nesprin$1 \alpha 2$ and the predominantly CNS-expressed giant isoform. ${ }^{5}$ In contrast, all but five SCAR8 (spinocerebellar ataxia, recessive, type 8)-associated truncating variants ${ }^{8}$ affect the giant and the large KASH-less nesprin-1 variant (KLNes1g) but leave nesprin-1 $\alpha 2$ intact (Figure 2 and Supplementary Table S1).,3,9-11 The five distal-truncating SCAR8 variants were compound heterozygous with truncating variants outside the nesprin- $1 \alpha 2$ region. ${ }^{12}$ The pathogenicity of SCAR8-associated variants is supported by finding variants in nesprin-1-interacting partners, spectrin- $\beta I I I$ and inositol 1,4,5-triphosphate receptor proteins, underlying other late-onset spinocerebellar ataxia phenotypes. ${ }^{13,14}$ SCAR8 patients can present with pure cerebellar atrophy, ataxia, and dysarthria, with variable age at onset of symptoms (6-50 years). ${ }^{9,12,15,16}$ More frequently, signs of upper and lower motor neuron disease, of brainstem dysfunction, or musculoskeletal abnormalities are variably present in SCAR 8 patients. ${ }^{12}$ Out of more than 80

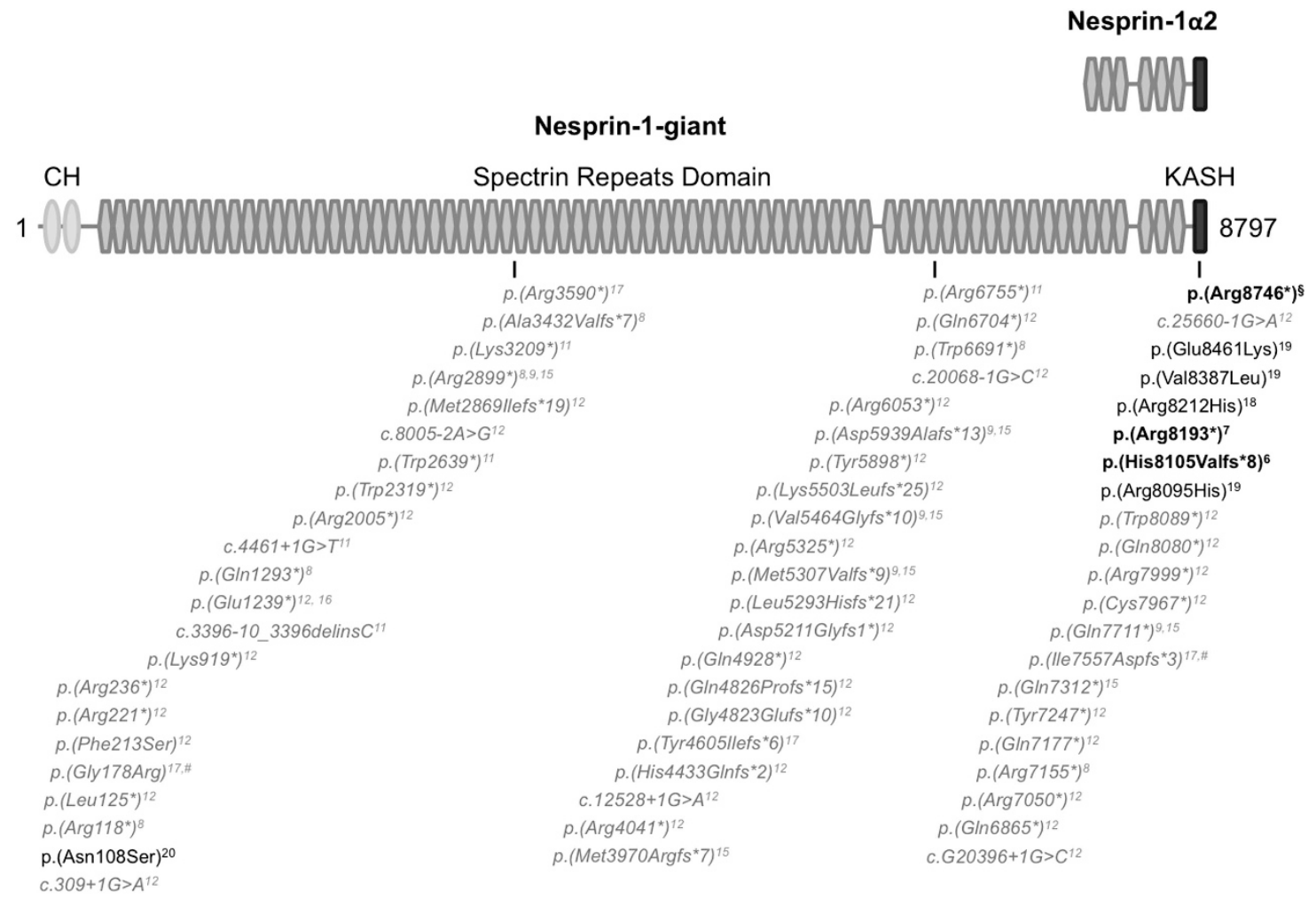

Figure 2 Nesprin-1 isoforms and human nesprin-1 variants. The two human nesprin-1 isoforms mainly expressed in cardiac and skeletal muscle are shown schematically. Nesprin-1 $\alpha 2$, one of the shortest nesprin-1 isoforms, is found almost exclusively in cardiac and skeletal muscle. Reported human diseasecausing nesprin-1 variants are compiled below the scheme. Italic gray letters denote bi-allelic SYNE1 variants that were identified in patients with cerebellar ataxia with slow progression accompanied by dysarthria and cerebellar atrophy (spinocerebellar ataxia, recessive, type 8 (SCAR8, ARCA-1; OMIM \#610743).8,9,11,12,15-17 Heterozygous SYNE1 missense variants shown in black letters were identified in patients with autosomal dominant Emery-Dreifuss muscular dystrophy, characterized by childhood-onset progressive muscle weakness and development of limb contractures (EDMD4; OMIM \#612998), ${ }^{19,20}$ and in patients with dilated cardiomyopathy. ${ }^{18}$ Bi-allelic truncating variants shown in bold black letters were identified in three families with recessively inherited myogenic AMC, associated with severe hypotonia from birth, delayed motor milestones, evolution of severe scoliosis and progressive motor decline after the first decade in one family, 6,7 and in this study. The variant nomenclature is based on NCBI reference sequence NP_892006.3, which represents the longest nesprin-1 isoform, and is a product of transcript NM_182961.3. CH, a pair of N-terminal CH domains that bind F-actin; KASH, Klarsicht/ANC-1/ Syne homology transmembrane domain that facilitates nuclear envelope localization. Disease-associated variants are compiled on the DNA level in Supplementary Table S1. \#Two variants on the same allele, §this study. 

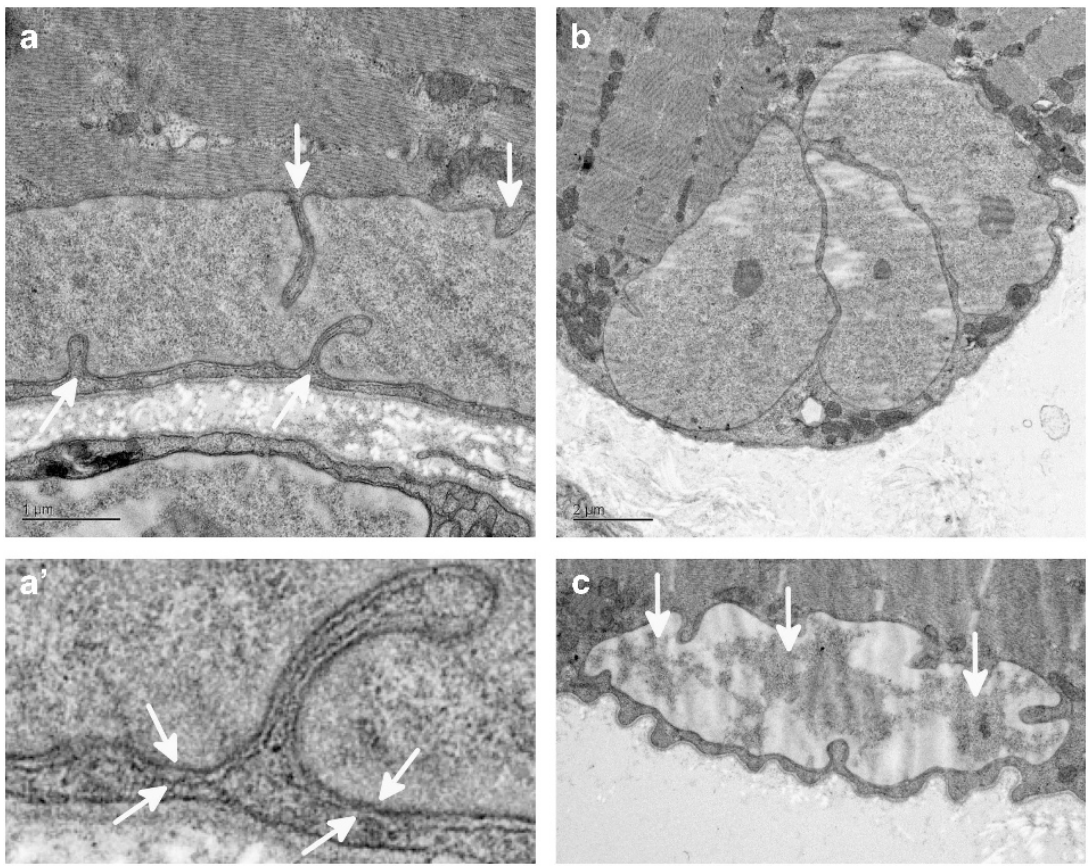

Figure 3 Perinuclear changes in skeletal muscle caused by homozygous nesprin-1 variant. (a) Multiple invaginations of the nuclear membrane. (a') Detail from a: detachment of the outer and inner nuclear membrane. (b) Multilobulated, honeycombed myonucleus. (c) Dystrophic appearance and abnormal condensation of the chromatin structure within a myonucleus.

reported SCAR8 patients, 4 exhibited respiratory failure and motor neuron involvement, with intellectual disability, and premature death at 36 and 44 years in 3 and 2 of these patients. ${ }^{12,17}$ The complex phenotype might be explained in one patient by the presence of a homozygous missense and a homozygous frame-shift variant c.[532G > C];[22668dup] (p.[Gly178Arg];[Ile7557Aspfs ${ }^{\star 3}$ ]) in SYNE1, which might affect distinct SYNE1 isoforms; it remains unexplained in a patient with a homozygous c.661C $>\mathrm{T}\left(\mathrm{p} .\left(\operatorname{Arg} 221^{\star}\right)\right)$ variant and in a sib-pair compound heterozygous for c.23995C $>\mathrm{T}$ (p.(Arg7999*)) and c. $20396+1 G>C$, respectively. ${ }^{12}$ Intriguingly, nesprin- $1 \alpha 2$ staining was reportedly reduced in muscle biopsies from three patients with homozygous truncating SYNE1 variants localizing outside the musclespecific isoform. ${ }^{4,12}$ Additional nesprin-1 isoforms and tissue-specific scaffolds might be affected by specific SYNE1 variants.

Gain-of-function effects on nesprin-1 were supposed to result from heterozygous missense variants near the KASH domain and in the $\mathrm{CH}$ domain in patients with dilative cardiomyopathy, ${ }^{18}$ and with autosomal dominant Emery-Dreifuss muscular dystrophy (EDMD4). ${ }^{19,20}$ The latter showed childhood-onset, progressive muscle weakness with development of limb contractures, with and without dilative cardiomyopathy. The pathogenicity of reported missense variants in EDMD4 was questioned, as rare SYNE1 variants were frequently identified in controls and in SCAR8 patients with bi-allelic truncating SYNE1 variants. ${ }^{12}$ The pathogenicity of private nesprin-1 variants as causing EDMD4 is supported in that variants in other components of the (peri)nuclear cytoskeleton, lamin $\mathrm{A} / \mathrm{C}$, and emerin are causes of EDMD and cardiomyopathies. Nesprin- $1 \alpha 2$ is enriched in cardiac, skeletal, and smooth muscle, ${ }^{21}$ and may provide mechanical strength to nuclei that are constantly exposed to contractile strain in muscle fibers. EDMD-associated nesprin-1 variants exhibited nuclear morphology defects and suggested defects of the 'linker of nucleoskeleton to cytoskeleton complex' (LINC). A disruption of LINC as causing
AMC is indicated by an enlarged perinuclear space in muscle cells in our patient (Figure 3). It is comparable to a separation of the inner and outer nuclear membrane resulting from siRNA knockdown of nesprin-1 in cell lines ${ }^{22}$ and the irregularly shaped nuclei with invaginations of the nuclear membrane with reduced heterochromatin at the nuclear membrane as observed in mice with a homozygous deletion of the nesprin-1 KASH domain. ${ }^{18,23}$ Honeycombing of myonuclei represented another morphologic pathology found in our patient's muscle biopsy. Light microscopy revealed a variation in size of muscle fibers without increased number of muscle fibers with central nuclei. Mice with a homozygous deletion of the nesprin-1 KASH domain display symptoms of the human EDMD and AMC phenotypes. They exhibit lethality with approximately half dying at or near birth from respiratory failure. Surviving mice display hindlimb weakness and an abnormal gait. With increasing age, kyphoscoliosis, muscle pathology, and cardiac conduction defects develop. ${ }^{23}$

Nesprin-1 variants can cause or are associated with a diverse spectrum of diseases including intellectual disability ${ }^{24}$ and bipolar disorder, ${ }^{25}$ which can be partly explained by the existence of diverse tissue-specific isoforms with scaffolding roles throughout the cell. In addition, variants in LINC components might act as disease modifiers in individuals with variants in EDMD genes. ${ }^{26}$ The homozygous truncating variant detected in our patient emphasizes the significance of the localization of the variant for the clinical phenotype and confirms that variants in SYNE1 cause autosomal recessive AMC and muscular weakness. As AMC is genetically heterogeneous, one should consider nesprin-1 variants as a possible cause.

\section{CONFLICT OF INTEREST}

The authors declare no conflict of interest. 
1 Randles KN, Lam le T, Sewry CA et al: Nesprins, but not sun proteins, switch isoforms at the nuclear envelope during muscle development. Dev Dyn 2010; 239: 998-1009.

2 Rajgor D, Shanahan CM: Nesprins: from the nuclear envelope and beyond. Expert Rev Mol Med 2013; 15: e5.

3 Duong NT, Morris GE, Lam le T et al: Nesprins: tissue-specific expression of epsilon and other short isoforms. PLOS ONE 2014; 9: e94380.

4 Mislow JM, Kim MS, Davis DB, McNally EM: Myne-1, a spectrin repeat transmembrane protein of the myocyte inner nuclear membrane, interacts with lamin A/C. J Cell Sci 2002; 115: 61-70.

5 Razafsky D, Hodzic D: A variant of Nesprin 1 giant devoid of KASH domain underlies the molecular etiology of autosomal recessive cerebellar ataxia type I. Neurobiol Dis 2015; 78: 57-67.

6 Attali R, Warwar N, Israel A et al: Mutation of SYNE-1, encoding an essential component of the nuclear lamina, is responsible for autosomal recessive arthrogryposis. Hum Mol Genet 2009; 18: 3462-3469.

7 Laquerriere A, Maluenda J, Camus A et al: Mutations in CNTNAP1 and ADCY6 are responsible for severe arthrogryposis multiplex congenita with axoglial defects. Hum Mol Genet 2014; 23: 2279-2289.

8 Noreau A, Bourassa CV, Szuto A et al: SYNE1 mutations in autosomal recessive cerebellar ataxia. JAMA Neurol 2013; 70: 1296-1331.

9 Gros-Louis F, Dupre N, Dion P et al: Mutations in SYNE1 lead to a newly discovered form of autosomal recessive cerebellar ataxia. Nat Genet 2007; 39: 80-85.

10 Cartwright S, Karakesisoglou I: Nesprins in health and disease. Semin Cell Dev Biol 2014; 29: 169-179.

11 Fogel BL, Lee H, Deignan JL et al: Exome sequencing in the clinical diagnosis of sporadic or familial cerebellar ataxia. JAMA Neurol 2014; 71: 1237-1246.

12 Synofzik M, Smets K, Mallaret M et al: SYNE1 ataxia is a common recessive ataxia with major non-cerebellar features: a large scale multi-centre study. Brain 2016; 139: 1378-1393.

13 Ikeda Y, Dick KA, Weatherspoon MR et al: Spectrin mutations cause spinocerebellar ataxia type 5. Nat Genet 2006: 38: 184-190.
14 van de Leemput J, Chandran J, Knight MA et al: Deletion at ITPR1 underlies ataxia in mice and spinocerebellar ataxia 15 in humans. PLoS Genet 2007; 3: e108.

15 Dupre N, Gros-Louis F, Chrestian N et al: Clinical and genetic study of autosomal recessive cerebellar ataxia type 1. Ann Neurol 2007; 62: 93-98.

16 Hamza W, Ali Pacha L, Hamadouche T et al: Molecular and clinical study of a cohort of 110 Algerian patients with autosomal recessive ataxia. BMC Med Genet 2015; 16: 36.

17 Izumi $\mathrm{Y}$, Miyamoto $\mathrm{R}$, Morino $\mathrm{H}$ et al: Cerebellar ataxia with SYNE1 mutation accompanying motor neuron disease. Neurology 2013; 80: 600-601.

18 Puckelwartz MJ, Kessler EJ, Kim G et al: Nesprin-1 mutations in human and murine cardiomyopathy. J Mol Cell Cardiol 2010; 48: 600-608.

19 Zhang Q, Bethmann C, Worth NF et al: Nesprin-1 and -2 are involved in the pathogenesis of Emery Dreifuss muscular dystrophy and are critical for nuclear envelope integrity. Hum Mol Genet 2007; 16: 2816-2833.

20 Fanin M, Savarese M, Nascimbeni AC et al: Dominant muscular dystrophy with a novel SYNE1 gene mutation. Muscle Nerve 2015; 51: 145-147.

21 Mislow JM, Holaska JM, Kim MS et al: Nesprin-lalpha self-associates and binds directly to emerin and lamin A in vitro. FEBS Lett 2002; 525: 135-140.

22 Zhang X, Xu R, Zhu B et al: Syne-1 and Syne-2 play crucial roles in myonuclear anchorage and motor neuron innervation. Development 2007; 134: 901-908.

23 Puckelwartz MJ, Kessler E, Zhang $\mathrm{Y}$ et al: Disruption of nesprin-1 produces an Emery Dreifuss muscular dystrophy-like phenotype in mice. Hum Mol Genet 2009; 18: 607-620.

24 Schuurs-Hoeijmakers JH, Vulto-van Silfhout AT, Vissers LE et al: Identification of pathogenic gene variants in small families with intellectually disabled siblings by exome sequencing. J Med Genet 2013; 50: 802-811.

25 Green EK, Grozeva D, Forty L et al: Association at SYNE1 in both bipolar disorder and recurrent major depression. Mol Psychiatry 2013; 18: 614-617.

26 Meinke P, Mattioli E, Haque F et al: Muscular dystrophy-associated SUN1 and SUN2 variants disrupt nuclear-cytoskeletal connections and myonuclear organization. PLoS Genet 2014; 10: e1004605. 